\title{
SOME REMARKS ON STRONG APPROXIMATION BY CESÀRO MEANS
}

\author{
HANS-JURGEN SCHMEISSER and WINFRIED SICKEL \\ Sektion Mathematik, Friedrich-Schiller-Universität \\ DDR-6900, Jena, Universitäts-Hochhaus \\ German Democratic Republic
}

\section{Introduction}

The problem under consideration here can be described as follows. If $f$ is a $2 \pi$-periodic function and if $(C, \alpha)_{v} f$ denotes the $v$ th Cesàro mean of order $\alpha$ of its Fourier series then we look for smoothness properties of $f$ which ensure that

$$
\sup _{x} \sum_{v=1}^{\infty} v^{s q}\left|\left(f-(C, \alpha)_{v} f\right)(x)\right|^{q}<\propto
$$

for some $q, 0<q<\infty$ and some real numbers $s$. Also we discuss the same problem if the sup-norm in (1.1) is replaced by the $L_{p}$-norm, $0<p<\infty$.

This is a problem of strong summability (approximation) for the means $(C, \alpha)_{v} f$, which is closely related to the periodic spaces $F_{p, q}^{s}(T)$ of TriebelLizorkin type. This was pointed out in [5]-[7] and in H. Triebel and H.-J. Schmeisser [8]. For the historical background and a comprehensive treatment of the case $p=\infty$ we refer to the recent book by L. Leindler [2].

In [5]-[7] the authors discussed such problems (also the inverse ones) for some classical approximation processes, e.g. for approximation by partial sums, Riesz-Bochner means, de la Vallée-Poussin means, and Abel-Poisson means. Results for $(C, \alpha)$-means cannot be derived immediately from the general results obtained in [6], [7] (cf. also [6], Remark 3).

Our aim is to close this gap. It turns out that the same ideas and tools apply to $(C, \alpha)$-means. The crucial point is inequality (3.19) below, a pointwiseestimate of a difference of Cesàro means.

In Section 2 we establish our results. The most interesting is the case of $p<1$. In contrast to [5]-[7] we only consider the one-dimensional case. In 
the $n$-dimensional case there are some open questions. The proofs are contained in Section 3.

The work was started during a stay at the Stefan Banach Mathematical Center in Warsaw in Spring 1986. We take the opportunity to thank our colleagues for their hospitality.

\section{The main result}

We start with some notation and definitions. Let $0<p<\infty$. As usual we put

$$
\begin{gathered}
\left\|f \mid L_{p}\right\|=\left(\int_{-\pi}^{\pi}|f(x)|^{p} d x\right)^{1 / p}, \\
\left\|f \mid L_{\infty}\right\|=\underset{-\pi \leqslant x \leqslant \pi}{\operatorname{ess} \sup _{-\pi}|f(x)|}
\end{gathered}
$$

for a measurable complex-valued $2 \pi$-periodic function $f$. The corresponding spaces are denoted by $L_{p}$. Furthermore, $D^{\prime}(T)$ denotes the set of all distributions on the torus $T . T$ is as usual represented by $[-\pi, \pi]$ (with $-\pi$ and $\pi$ identified). The Fourier coefficients of $f \in D^{\prime}(T)$ are given by

$$
\hat{f}(k)=\frac{1}{2 \pi} f\left(e^{-i k x}\right), \quad k=0, \pm 1, \pm 2, \ldots
$$

We now define the Cesàro means of order $\alpha>0$ of a periodic distribution $f \in D^{\prime}(T)$. We put

$$
(C, \alpha)_{v} f(x)=\frac{1}{A_{v}^{\alpha}} \sum_{k=-v}^{v} A_{v-|k|}^{\alpha} \hat{f}(k) e^{i k x}, \quad x \in[-\pi, \pi], v=0,1, \ldots,
$$

where

$$
A_{v}^{\alpha}=\left(\begin{array}{c}
v+\alpha \\
v
\end{array}\right)=\frac{\Gamma(v+\alpha+1)}{\Gamma(v+1) \Gamma(\alpha+1)} .
$$

The means $(C, \alpha)_{v} f$ can be rewritten in the following way. There exists a function $\Omega_{\alpha}$ defined on $R_{+}=\{x \mid x \geqslant 0\}$ with the properties (cf. J. J. Gergen [1], W. Trebels [10]):

(i) $\Omega_{\alpha}$ and its derivatives $\frac{d \Omega_{\alpha}}{d x}, \ldots, \frac{d^{l} \Omega_{\alpha}}{d x^{l}}, \ldots$ are continuous on $R_{+}$with

$$
\Omega_{\alpha}(0)=\frac{d \Omega_{\alpha}}{d x}(0)=\ldots=\frac{d^{l} \Omega_{\alpha}}{d x^{l}}(0)=\ldots=0
$$

(ii) We have

$$
\Omega_{\alpha}(x)=\frac{\Gamma(\alpha+x)}{\Gamma(x+1) \Gamma(\alpha+1)}, \quad x \geqslant 1
$$


(iii) There exists a continuous function $u_{\alpha}$ defined on $\boldsymbol{R}_{+}$and a positive constant $c_{\alpha}$ such that

$$
\begin{gathered}
\Omega_{\alpha}(x)=\int_{0}^{x}(x-t)^{\alpha} u_{\alpha}(t) d t, \quad x \in R_{+}, \\
\left|u_{\alpha}(t)\right| \leqslant c_{\alpha}\left(\frac{1}{1+t^{2}}\right), \quad t \in R_{+} .
\end{gathered}
$$

(iv) There exist positive constants $c_{0}, c_{1}, c_{2}$ such that

$$
c_{1} x^{\alpha-l} \leqslant\left|\frac{d^{l} \Omega_{\alpha}}{d x^{l}}(x)\right| \leqslant c_{2} x^{\alpha-l}, \quad l=0,1, \ldots,[\alpha], \text { if } x \geqslant c_{0} .
$$

Using $\Omega_{\alpha}$ we have

$$
(C, \alpha)_{v} f(x)=\sum_{k=-v}^{v} \frac{\Omega_{\alpha}(v-|k|+1)}{\Omega_{\alpha}(v+1)} \hat{f}(k) e^{i k x}, \quad x \in[-\pi, \pi], v=0,1, \ldots
$$

For abbreviation we introduce the functions $\psi_{v}^{\alpha}$ by

$$
\psi_{v}^{\alpha}(x)= \begin{cases}\frac{\Omega_{\alpha}(v-|x|+1)}{\Omega_{\alpha}(v+1)}, & 0 \leqslant|x| \leqslant v+1, \\ 0, & \text { otherwise. }\end{cases}
$$

This leads to

$$
(C, \alpha)_{v} f(x)=\sum_{k=-\infty}^{\infty} \psi_{\nu}^{\alpha}(k) \hat{f}(k) e^{j k x}, \quad x \in[-\pi, \pi], v=0,1, \ldots,
$$

which is more convenient for us than (2.1).

Next we define the spaces $F_{p, q}^{s}(T)$ and $B_{p, q}^{s}(T)$. Let $\varphi \in C^{\infty}(R)$ be a function with

$$
\operatorname{supp} \varphi \subset\{\xi|1 / 2 \leqslant| \xi \mid \leqslant 2\} \quad \text { and } \quad \sum_{j=0}^{\infty} \varphi\left(2^{-j} \xi\right)=1 \quad \text { for }|\xi| \geqslant 1
$$

We put .

$$
\varphi_{l}(\cdot)= \begin{cases}\varphi\left(2^{-l} \cdot\right), & l=0,1, \ldots, \\ 0, & l=-1,-2, \ldots\end{cases}
$$

Definition. Let $-\infty<s<\infty$ and $0<q<\infty$.

(i) If $0<p<\infty$ then

$$
\begin{aligned}
& F_{p, q}^{s}(T)=\left\{f \mid f \in D^{\prime}(T),\right. \\
& \left.\quad\left\|f\left|F_{p, q}^{s}(T)\|=\|\left(\sum_{j=0}^{\infty} 2^{j s q}\left|\sum_{k=-\infty}^{\infty} \varphi_{j}(k) \hat{f}(k) e^{i k x}\right|^{q}\right)^{1 / q}\right| L_{p}\right\|+|\hat{f}(0)|<\infty\right\},
\end{aligned}
$$




$$
\begin{aligned}
& F_{p, \infty}^{s}(T)=\left\{f \mid f \in D^{\prime}(T)\right. \\
& \left.\qquad\left\|f\left|F_{p, \infty}^{s}(T)\|=\| \sup _{j=0,1, \ldots} 2^{j s}\right| \sum_{k=-\infty}^{\infty} \varphi_{j}(k) \hat{f}(k) e^{i k x}|| L_{p}\right\|+|\hat{f}(0)|<\infty\right\} .
\end{aligned}
$$

(ii) If $0<p \leqslant \infty$ then

$$
\begin{aligned}
& B_{p, q}^{s}(T)=\left\{f \mid f \in D^{\prime}(T),\right. \\
& \left\|f\left|B_{p, q}^{s}(T) \|=\left(\sum_{j=0}^{\infty} 2^{j s q}\left\|\sum_{k=-\infty}^{\infty} \varphi_{j}(k) \hat{f}(k) e^{i k x} \mid L_{p}\right\|^{q}\right)^{1 / q}+\right| \hat{f}(0) \mid<\infty\right\}, \\
& B_{p, \infty}^{s}(T)=\left\{f \mid f \in D^{\prime}(T),\right. \\
& \left.\left\|f\left|B_{p, \infty}^{s}(T)\left\|=\sup _{j=0,1, \ldots} 2^{j s}\right\| \sum_{k=-\infty}^{\infty} \varphi_{j}(k) \hat{f}(k) e^{i k x}\right| L_{p}\right\|+|\hat{f}(0)|<\infty\right\} .
\end{aligned}
$$

Remark 1. In the literature the spaces defined above are called TriebelLizorkin and Besov spaces. They are quasi-Banach spaces and are independent of the choice of $\varphi$. Furthermore, we have the embeddings

$$
F_{p, q}^{s}(T) \subset L_{p} \cap L_{1} \quad \text { and } \quad B_{p, q}^{s}(T) \subset L_{p} \cap L_{1}
$$

if $s>\max (0,1 / p-1)$. More information about Triebel-Lizorkin and Besov spaces on the torus or on $R_{n}$, such as characterizations by differences and derivatives, characterizations by approximation, or relations to more classical function spaces, can be found in H. Triebel [11], H.-J. Schmeisser and H. Triebel [8], or H.-J. Schmeisser [4].

Now we state our main result.

TheOREM. Let $\alpha>0$ and $0<s<1$.

(i) Let $1 /(\alpha+1)<p<\infty$ and $1 /(\alpha+1)<q \leqslant \infty$. If $f \in L_{p} \cap L_{1}$ then

$$
\begin{array}{r}
\left\|f\left|L_{p}\|+\|\left(\sum_{v=1}^{\infty} v^{s q-1}\left|\left(f-(C, \alpha)_{v} f\right)(x)\right|^{q}\right)^{1 / q}\right| L_{p}\right\|<\infty \Leftrightarrow f \in F_{p, q}^{s}(T) \\
\left(\left\|f\left|L_{p}\|+\| \sup _{v} v^{s}\right|\left(f-(C, \alpha)_{v} f\right)(x)|| L_{p}\right\|<\infty \text { if } q=\infty\right) .
\end{array}
$$

(ii) Let $1 /(\alpha+1)<p \leqslant \infty$ and $0<q \leqslant \infty$. If $f \in L_{p} \cap L_{1}$ then

$$
\begin{aligned}
\left\|f \mid L_{p}\right\|+\left(\sum_{v=1}^{\infty} v^{s q-1}\left\|\left(f-(C, \alpha)_{v} f\right)(x) \mid L_{p}\right\|^{q}\right)^{1 / q}<\infty \Leftrightarrow f \in B_{p, q}^{s}(T) \\
\left(\left\|f\left|L_{p}\left\|+\sup _{v} v^{s}\right\|\left(f-(C, \alpha)_{v} f\right)(x)\right| L_{p}\right\|<\infty \text { if } q=\infty\right) .
\end{aligned}
$$

(iii) If $0<q<\infty$ and $0<u<\infty$ then 


$$
\begin{array}{r}
\left\|f \mid L_{\infty}(T)\right\|+\left(\sum _ { j = 0 } ^ { \infty } \left\|_{v=2^{j}}^{2^{j+1}-1} v^{s u-1}\left|\left(f-(C, \alpha)_{v} f\right)(x)\left\|^{u} \mid L_{\infty}(T)\right\|^{\mid / \mu}\right)^{1 / q}<\infty\right.\right. \\
\Leftrightarrow f \in B_{\infty, q}^{s}(T) .
\end{array}
$$

Remark 2. The parts $\Rightarrow$ (the so-called inverse results) can be sharpened. (i)-(iii) remain true if $0<p, q<\infty$ and $s>0$ (cf. [6], Theorems 1,2 and Remark 3). Moreover, the expressions on the left-hand sides of $(2.10)-(2.12)$ are equivalent quasi-norms in the corresponding spaces on the right-hand sides.

Corollary. Let $0<s<1$ and $0<q<\infty$.

(i) If $f \in L_{\infty}(T)$ then

$$
\left\|\sum_{v=1}^{\infty} v^{s q-1}\left|\left(f-(C, \alpha)_{v} f\right)(x)\right|^{q} \mid L_{\infty}(T)\right\|<\infty
$$

implies $f \in B_{\infty, \infty}^{s}(T)$.

(ii) If $f \in B_{\infty, q}^{s}(T)$ then

$$
\left\|\sum_{v=1}^{\infty} v^{s q-1}\left|\left(f-(C, \alpha)_{v} f\right)(x)\right|^{q} \mid L_{\infty}(T)\right\|<\infty .
$$

Remark 3. (i) is stated in [6], Corollary 1 and Remark 6. To prove (ii) one can follow the same lines as in [6], Corollary 3 and Remark 12. Moreover, as pointed out in [9], part (i) of our corollary can be sharpened. If $1<q<\infty$ the finiteness of the expression in (2.13) implies $f \in F_{\infty, q}^{s}(T)$. Here $F_{\alpha, q}^{s}(T)$ means the dual space of $F_{1, q^{\prime}}^{-s}(T)$, where $1 / q+1 / q^{\prime}=1$. Note that

$$
B_{\infty, q}^{s}(T) \subset F_{\infty, q}^{s}(T) \subset B_{\infty, \infty}^{s}(T)
$$

and both embeddings are proper (cf. H.-J. Schmeisser and H. Triebel [8], Chapter 3).

Remark 4. We give some comments on the parameter ranges in our theorem. If $p \geqslant 1$ then the restriction of $s$ to the values less than 1 is quite natural. The case $s=1$ corresponds to the saturation order of Cesàro approximation (cf. W. Trebels [10], Theorem 4.9). It follows that

$$
\text { - }\left\|\left(f-(C, \alpha)_{v} f\right)(x) \mid L_{p}\right\|=o\left(v^{-1}\right), \quad v \rightarrow \infty,
$$

implies $f \equiv c$ if $1 \leqslant p \leqslant \infty$. In case $0<p<1$ such a final answer seems to be unknown. The restriction of $1 / p$ to the values less than $\alpha+1$ also appears in the work of P. Oswald [3] in connection with the boundedness properties of the maximal operator

$$
(T f)(x)=\sup _{v}\left|(C, \alpha)_{v} f(x)\right|
$$


in some subspaces of $F_{p, 2}^{0}(T)$. Furthermore, in the case of approximation by Riesz-Bochner means of order $\alpha$ we have the same bound for $1 / p$ (cf. [6], Theorem 8).

Remark 5. The theory developed in [6] and [7] works in any dimension. A consequence of this fact is the correctness of the inverse results $(\Rightarrow)$ in any dimension. Our direct results are restricted to the case $n=1$, and extension to $n>1$ seems to be open. We return to this problem in connection with Lemma 1 in the following section.

\section{Proofs}

To prove our main result we need some lemmata. Let $B_{p, q}^{s}(R)$ be the nonperiodic counterpart of the spaces $B_{p, q}^{s}(T)$ (cf. H. Triebel [11], H.-J. Schmeisser [4]).

Lemma 1. Let $\alpha>-1$. Let $\tau$ be a real number with $0 \leqslant \tau \leqslant 2$. Let $\chi$ be $a$ $C^{\infty}$-function with $\chi(x)=1$ if $0 \leqslant x \leqslant 4$ and $\chi(x)=0$ if $x \in R \backslash[-1,5]$. Then

$$
f_{\tau}^{\alpha}(x):=(\tau-x)^{\alpha}+\chi(x)= \begin{cases}(\tau-x)^{\alpha} \chi(x), & \tau \geqslant x, \\ 0 & \text { otherwise, }\end{cases}
$$

is a uniformly bounded family of functions in $B_{1, \infty}^{1+\alpha}(R)$.

Proof. First one checks that $f_{\tau}^{\alpha}$ is a uniformly bounded family in $W_{1}^{m}(R)$ if $\alpha-m>-1$ (here $W_{1}^{m}(R)$ denotes the usual Sobolev space on the real line).

Let $0>\delta>-1$. We have

$$
\int_{|x-\tau| \leqslant 2|h|}\left|\Delta_{h}^{1} f_{\tau}^{\delta}(x)\right| d x \leqslant c|h|^{1+\delta}
$$

and by the mean value theorem

$$
\int_{k|h| \leqslant|x-\tau| \leqslant(k+1)|h|}\left|\Delta_{h}^{1} f_{\tau}^{\delta}(x)\right| d x \leqslant c|h|^{2}(k|h|)^{\delta-1}=c|h|^{1+\delta} k^{\delta-1}
$$

for any $h \in R$ and any $k, k=2,3, \ldots$ The last formula leads to

$$
\begin{aligned}
\int_{|x-\tau|>2|h|}\left|\Delta_{h}^{1} f_{\tau}^{\delta}(x)\right| d x & \leqslant \sum_{k=2}^{3([1 / h]+1)} \int_{k|h| \leqslant|x-\tau| \leqslant(k+1)|h|}\left|\Delta_{h}^{1} f_{\tau}^{\delta}(x)\right| d x \\
& \leqslant c|h|^{1+\delta} \sum_{k=2}^{\infty} k^{\delta-1} \leqslant c|h|^{1+\delta} .
\end{aligned}
$$

(3.1) and (3.2) prove that $f_{\tau}^{\delta}$ is a uniformly bounded family in $B_{1, \infty}^{1+\delta}(R)$.

By observing that the $([\alpha]+1)$-th derivative of $f_{\tau}^{\alpha}$ is a function of the type of $f_{\tau}^{\delta}$, the lemma is proved for any noninteger $\alpha$. But if $\alpha$ is a 
nonnegative integer one checks easily that the derivative of order $\alpha$ is the product of the characteristic function of an interval and a smooth function and hence it belongs to $B_{1, \infty}^{1}(R)$ (cf. $H$. Triebel [11], 2.8). This completes the proof.

Remark 6. One can also prove a radial-symmetric version of the lemma in dimension $n$ if one modifies $\chi$ in such a wav that $0 \notin \operatorname{supp} \chi$. But unfortunately the smoothness of such an extended function does not depend on the dimension. If $n>1$ this leads in the following to unnatural restrictions on the parameter $p$.

Let $\Phi \in C^{\infty}(R)$ be a function with

$$
\Phi(x)=1 \text { if } 1 / 2 \leqslant|x| \leqslant 2 \text { and } \operatorname{supp} \Phi \subset\{x|1 / 4 \leqslant| x \mid \leqslant 4\} .
$$

Let $\alpha>0$ be fixed. We put

$$
\gamma_{v, l}(x):=\frac{1-\psi_{v}^{\alpha}\left(2^{j+1} x\right)}{|x|} \Phi\left(2^{-1+1} x\right)
$$

LeMMA 2. If $j=0,1, \ldots, 2^{j} \leqslant v<2^{j+1},-j \leqslant l \leqslant 1$, then $\gamma_{v, l} \in B_{1, \infty}^{\alpha+1}(R)$. Furthermore, there exists a positive constant $c$ independent of $j, v$, and $l$ such that

$$
\left\|\gamma_{v, l} \mid B_{1, \infty}^{1+\alpha}(R)\right\| \leqslant c 2^{-\alpha l}
$$

Proof. Step 1. We estimate $\left\|\gamma_{v, l} \mid L_{1}(R)\right\|$.

Let $j_{0}$ be an integer with $2^{j_{0}-1} \geqslant c_{0}$, where $c_{0}$ is the number from (2.6). Let $j \geqslant j_{0}$ and let $r \in R_{+}$be such that $2^{j+1} r \leqslant v / 2$. As a consequence of (2.6) and (2.7) we obtain by the mean value theorem

$$
\left|\frac{1-\psi_{v}^{\alpha}\left(2^{j+1} r\right)}{r}\right|=\left|\frac{\Omega_{\alpha}(v+1)-\Omega_{\alpha}\left(v+1-2^{j+1} r\right)}{r \Omega_{\alpha}(v+1)}\right|=\left|\frac{\frac{d \Omega_{\alpha}}{d r}(\xi) \cdot 2^{j+1} r}{r \Omega_{\alpha}(v+1)}\right| \leqslant c
$$

for $v / 2+1 \leqslant \xi \leqslant v+1$. Here the constant $c$ is independent of $j$ and $v$. On the other hand, if $2^{j+1} r \geqslant v / 2$, then $r \geqslant 1 / 4$ and hence

$$
\left|\frac{1-\psi_{v}^{\alpha}\left(2^{j+1} r\right)}{r}\right| \leqslant 4 \text {. }
$$

Now, (3.6) and (3.7) together with the properties of $\Phi$ lead to the estimate

$$
\sup _{\substack{j \geqslant j_{0} \\ j \leqslant v<2^{j+1} \\-j \leqslant l \leqslant 1}} 2^{-l}\left\|\gamma_{v, l} \mid L_{1}(R)\right\| \leqslant c .
$$

Step 2. We estimate the derivatives of $\gamma_{v, l}$.

Clearly, $\gamma_{v, l} \in C^{\infty}(R)$ and hence the following is meaningful. For any

24 - Banach Center t. 22 
positive integer $m$ and $x \geqslant 0$ we have

$$
\begin{aligned}
\left|\frac{d^{m} \gamma_{v, l}}{d x^{m}}(x)\right| \leqslant & c \sum_{\substack{0 \leqslant m_{1}, m_{2} \leqslant m \\
m_{1}+m_{2}=m}}\left|\left(1-\psi_{v}^{\alpha}\left(2^{j+1} x\right)\right) \frac{2^{-l m_{2}}}{x^{m_{1}+1}} \frac{d^{m_{2}} \Phi}{d x^{m_{2}}}\left(2^{-l+1} x\right)\right| \\
+ & \sum_{\substack{0 \leqslant m_{1}, m_{2} \leqslant m \\
1 \leqslant m_{3} \leqslant m \\
m_{1}+m_{2}+m_{3}=m}} \frac{2^{j m_{3}}}{\Omega_{\alpha}(v+1)} \\
& \times \frac{d^{m_{3}} \Omega_{\alpha}}{d x^{m_{3}}}\left(\left(v+1-2^{j+1}|x|\right)_{+}\right) \frac{2^{-l m_{2}}}{x^{m_{1}+1}} \frac{d^{m_{2}} \Phi}{d x^{m_{2}}}\left(2^{-l+1} x\right) \mid .
\end{aligned}
$$

Now, to estimate the derivatives we distinguish two cases.

First we consider all integers $l$ with $-j \leqslant l \leqslant-3$. Hence, if $x \in \operatorname{supp} \Phi\left(2^{-1+1} \cdot\right)$ we have $v+1-2^{j+1} x \geqslant 2^{j-1}$. Using again (2.6) with $j$ $\geqslant j_{0},(3.7)$, and the conditions on the support of $\Phi$ one obtains in this case

$$
\begin{aligned}
\left|\frac{d^{m} \gamma_{v, l}}{d x^{m}}(x)\right| \leqslant c\left|1-\psi_{v}^{\alpha}\left(2^{j+1} x\right)\right| 2^{-l(m+1)} & \\
& +\sum_{1 \leqslant m_{3} \leqslant m} 2^{j\left(m_{3}-\alpha\right)}\left|\frac{d^{m_{3}} \Omega_{\alpha}}{d x^{m_{3}}}\left(\left(v+1-2^{j+1}|x|\right)_{+}\right)\right| 2^{-l\left(m-m_{3}+1\right)} \\
\leqslant & c 2^{-l m},
\end{aligned}
$$

where $c$ is independent of $j \geqslant j_{0}, 2^{j} \leqslant v<2^{j+1}$ and $-j \leqslant l \leqslant-3$.

If $-2 \leqslant l \leqslant 1$ then $2^{j-4} \leqslant 2^{j+1} x \leqslant 2^{j+2}$ for $x \in \operatorname{supp} \Phi\left(2^{-l+1} \cdot\right)$. The restriction of $m$ to the values with $\alpha-m>1$ allows us to use the integral representation of the derivative of $\Omega_{\alpha}$ (cf. (2.5)). Using again (3.9) and (3.10) we obtain for such values of $l$ and $m$

$$
\begin{aligned}
& \text { 1) } \quad \int\left|\frac{d^{m} \gamma_{v, l}}{d x^{m}}(x)\right| d x \\
& \leqslant c\left(1+\sum_{1 \leqslant m_{3} \leqslant m} 2^{j\left(m_{3}-\alpha\right)} 2^{-l\left(m-m_{3}+1\right)}\right) \int_{2}^{4}\left|\frac{d^{m_{3}} \Omega_{\alpha}}{d x^{m_{3}}}\left(\left(v+1-2^{j+1}|x|\right)+\right)\right| d x .
\end{aligned}
$$

Because

$$
\begin{aligned}
\int_{-5}^{4}\left|\frac{d^{m} \Omega_{\alpha}}{d x^{m}}\left(\left(v+1-2^{j+1}|x|\right)_{+}\right)\right| d x & \leqslant c 2^{-j} \int_{0}^{v+1} \int_{2^{j-4}}^{v+1}(v+1-\tau-t)_{+}^{\alpha-m}\left|u_{\alpha}(t)\right| d \tau d t \\
& \leqslant c 2^{-j} \int_{0}^{v+1}(v+1-t)^{\alpha-m+1}\left|u_{\alpha}(t)\right| d t \\
& \leqslant c 2^{j(\alpha-m)} \quad \text { if } \alpha-m>-1,
\end{aligned}
$$


it follows from (3.11), (3.10), and (3.8) that

$$
\sup _{\substack{j \geqslant j_{0} \\ 2^{j} \leqslant v<2^{j+1} \\-j \leqslant l \leqslant 1}} 2^{-l(1-m)}\left\|\gamma_{v, l} \mid W_{1}^{m}(R)\right\| \leqslant c .
$$

If $\alpha$ is an integer then $\Omega_{\alpha}$ is a polynomial of degree $\alpha$ for $x \geqslant 1$. Hence $\frac{d^{\alpha+1} \Omega_{\alpha}}{d x^{\alpha+1}}(x)=0$ for $x \geqslant 1$. Using

$$
\int_{2}^{4}\left|\frac{d^{\alpha+1} \Omega_{\alpha}}{d x^{\alpha+1}}\left(\left(v+1-2^{j+1}|x|\right)_{+}\right)\right| d x \leqslant c 2^{-j} \int_{v}^{v+1}\left|\frac{d^{\alpha+1} \Omega_{\alpha}}{d x^{\alpha+1}}(v+1-\tau)\right| d \tau \leqslant c 2^{-j}
$$

we see that (3.12) is true if $\alpha-m \geqslant-1$, too. The continuous embedding $W_{1}^{m}(R) \subset B_{1, \infty}^{m}(R)$ proves the lemma if $\alpha$ is an integer.

Step 3. Let $\alpha$ be noninteger. Let $-j \leqslant l \leqslant-3$ and $0<\theta<1$. In Step 2 we have proved

$$
\left\|\gamma_{v, l} \mid W_{1}^{m}(R)\right\| \leqslant c_{m} 2^{l(1-m)}
$$

for any nonnegative $m$. Since

$$
\left(B_{1, \infty}^{0}(R), B_{1, \infty}^{m}(R)\right)_{\theta, \infty}=B_{1, \infty}^{m(1-\theta)}(R)
$$

we have the inequality

$$
\left\|\gamma_{v, l} \mid B_{1, \infty}^{m(1-\theta)}(R)\right\| \leqslant c 2^{l \theta} 2^{l(1-m)(1-\theta)}=c 2^{l(1-m(1-\theta))}
$$

where $c$ is independent of $\gamma_{v, l}$. This proves the lemma under the restrictions $j \geqslant j_{0}$ and $-j \leqslant l \leqslant-3$.

Let $-2 \leqslant l \leqslant 1$. Note that the support of $\gamma_{v, l}$ is contained in $\left\{x\left|2^{-5} \leqslant\right| x \mid \leqslant 4\right\}$. Restricted to this domain, functions of the type $\frac{d^{m_{1}} \Phi}{d x^{m_{1}}}(\tau x):|x|^{-m_{2}}$ are pointwise multipliers for $B_{1, q}^{s}(R)$ spaces (cf. H. Triebel $[11], 2.8)$. Let $\chi$ be the function of Lemma 1. Then

(3.14) $\quad\left\|\gamma_{v, l} \mid B_{1, \infty}^{\alpha+1}(R)\right\|$

$$
\begin{aligned}
\leqslant c\left\|\frac{d^{[\alpha]+1} \gamma_{v, l}}{d x^{[\alpha]+1}} \mid B_{1, \infty}^{\alpha-[\alpha]}(R)\right\| \leqslant c\left(\left\|\chi(x)\left(1-\psi_{v}^{\alpha}\left(2^{j+1} x\right)\right) \mid B_{1, \infty}^{\alpha-[\alpha]}(R)\right\|\right) \\
\left.+\sum_{1 \leqslant m \leqslant[\alpha]+1} 2^{j(m-\alpha)}\left\|\frac{d^{m} \Omega_{\alpha}}{d x^{m}}\left(\left(v+1-2^{j+1}|x|\right)_{+}\right) \chi(x) \mid B_{1, \infty}^{\alpha-[\alpha]}(R)\right\|\right) .
\end{aligned}
$$


Using (2.5) and Lemma 1 we obtain

$$
\begin{aligned}
\| \frac{d^{m} \Omega_{\alpha}}{d x^{m}}((v+ & \left.\left.1-2^{j+1}|x|\right)_{+}\right) \chi(x) \mid B_{1, \infty}^{\alpha-[\alpha]}(R) \| \\
& \leqslant c\left\|\chi(x) \int_{0}^{\infty}\left(v+1-2^{j+1}|x|-t\right)_{+}^{\alpha-m} u_{\alpha}(t) d t \mid B_{1, \infty}^{\alpha-[a]}(R)\right\| \\
& \leqslant c \sup _{0<t<v+1}\left\|\chi(x)\left(v+1-2^{j+1}|x|-t\right)_{+}^{\alpha-m} \mid B_{1, \infty}^{\alpha-[\alpha]}(R)\right\| \\
& \leqslant c 2^{j(\alpha-m)} \sup _{0<t<v+1}\left\|\chi(x)\left(\frac{v+1-t}{2^{j+1}}-|x|\right)_{+}^{a-m} \mid B_{1, \infty}^{\alpha-[a]}(R)\right\| \\
& \leqslant c 2^{j(\alpha-m)}
\end{aligned}
$$

for any nonnegative $m$ with $\alpha-m>-1$. Because

$$
\Delta_{h}^{1}\left(1-\psi_{v}^{\alpha}\left(2^{j+1} x\right)\right)=\Delta_{h}^{1} \psi_{v}^{\alpha}\left(2^{j+1} x\right)=\frac{1}{\Omega_{\alpha}(v+1)} \cdot \Delta_{h}^{1} \Omega_{\alpha}\left(\left(v+1-2^{j+1}|x|\right)_{+}\right)
$$

we have by (3.15) and (3.8)

$$
\left\|\chi(x)\left(1-\psi_{v}^{\alpha}\left(2^{j+1} x\right)\right) \mid B_{1, \infty}^{\alpha-[\alpha]}(R)\right\| \leqslant c .
$$

Putting this estimate into (3.14) and taking into account that the constants do not depend on $j \geqslant j_{0}$ and $v, 2^{j} \leqslant v<2^{j+1}$, we get the counterpart of (3.13) with $-2 \leqslant l \leqslant 1$, at least if $m(1-\theta)=\alpha+1$.

This proves the lemma.

The next and last lemma we wish to formulate is more or less a modification of Lemma 3 in [6] and so we omit the proof (cf. also Corollary 2 in [6]).

As usual, $M f$ denotes the Hardy-Littlewood maximal function of an integrable function $f$.

Lemma 3. Let $0<r \leqslant 1$ and $1 / r<\lambda<\infty$. Let $\gamma \in B_{1, \infty}^{\lambda}(R)$ and

$$
\operatorname{supp} \gamma \subset\left\{x|| x \mid \leqslant 2^{l}\right\}, \quad l=0, \pm 1, \pm 2, \ldots
$$

Let $j=0,1, \ldots$ If

$$
f_{m}(x)=\sum_{|k| \leqslant 2^{m}} a_{k} e^{i k x}, \quad x \in T, m=0,1, \ldots,
$$

then there exists a constant $c$ independent of $f_{m}, \gamma, j, l$, and $x$ such that

$$
\left|\sum_{k=-\infty}^{\infty} \gamma\left(2^{-j} k\right) \hat{f}_{m}(k) e^{i k x}\right| \leqslant c 2^{(\tau-j)(1 / r-1)}\left\|\gamma \mid B_{1, \infty}^{\lambda}(R)\right\|\left(M\left|f_{m}\right|^{1 / r}(x)\right.
$$

where $\tau:=\max (l+j, m)$. 
Now we are in a position to give the proof of the theorem.

Proof of the theorem. We prove (i). In principle we follow [6]. Let $f \in F_{p, q}^{s}(T) \cap L_{p}, 0<p, q<\infty$, and $s>0$. Let $0<r<\min (1, p, q)$. Analogously to [6], (4.6), we obtain

$$
\begin{aligned}
& \sum_{v=1}^{\infty} v^{\varepsilon q-1}\left|\left(f-(C, \alpha)_{v} f\right)(x)\right|^{q} \\
& \qquad c \sum_{j=0}^{\infty} 2^{j s q} \sup _{2^{j} \leqslant v<2^{j+1}}\left|\left((C, \alpha)_{2^{j+1}} f-(C, \alpha)_{v} f\right)(x)\right|^{q}
\end{aligned}
$$

where $c$ is independent of $f$ and $x \in T$. Now we define a family of functions by

$$
\eta_{j, v}^{\alpha}(x)=\psi_{2^{j+1}}^{\alpha}(x)-\psi_{v}^{\alpha}(x), \quad x \in R, j=0,1, \ldots, \text { and } 2^{j} \leqslant v<2^{j+1} .
$$

We can write

$$
\left((C, \alpha)_{2^{j+1}} f-(C, \alpha)_{v} f\right)(x)=\sum_{k=-\infty}^{\infty} \eta_{j, v}^{\alpha}(k) \hat{f}(k) e^{i k x}, \quad x \in T .
$$

Furthermore, it is not hard to see that the identity

$$
\eta_{j, v}^{a}(k)=\sum_{l=-\infty}^{1} \eta_{j, v}^{a}(k) \varphi_{l+j}(k) \Phi_{l+j}(k)
$$

holds (here $\varphi_{1+j}$ are the functions defined in (2.9) and

$$
\Phi_{j}(\cdot)= \begin{cases}\Phi\left(2^{-j}\right), & j=0,1,2, \ldots, \\ 0, & j=-1,-2, \ldots,\end{cases}
$$

where $\Phi$ is defined in (3.3)). Now we put

$$
f_{l}(x)=\sum_{k=-\infty}^{\infty}\left|2^{-l-1} k\right| \varphi_{l}(k) \hat{f}(k) e^{i k x}, \quad x \in T, l=0, \pm 1, \pm 2, \ldots
$$

Analogously to [6], (4.10), we obtain

$$
\begin{aligned}
\left|\sum_{k=-\infty}^{\infty} \eta_{j, v}^{\alpha}(k) \hat{f}(k) e^{i k x}\right| & \\
& \leqslant\left.\sum_{l=-\infty}^{1} 2^{l}\left|\sum_{k=-\infty}^{\infty}\right| 2^{-j-1} k\right|^{-1} \eta_{j, v}^{\alpha}(k) \Phi_{j+l}(k) \hat{f}_{j+l}(k) e^{i k x} \mid .
\end{aligned}
$$

Obviously, we have

$$
\frac{\eta_{j, v}^{\alpha}(k) \Phi_{j+l}(k)}{\left|2^{-j-1} k\right|}=\gamma_{v, l}\left(2^{-(j+1)} k\right)-\gamma_{2^{j+1, l}}\left(2^{-(j+1)} k\right) .
$$


On applying Lemmas 2 and 3, (3.18) leads to

$$
\begin{aligned}
\left|\sum_{k=-\infty}^{\infty} \eta_{j, v}^{\alpha}(k) \hat{f}(k) e^{i k x}\right| & \leqslant c \sum_{l=-j}^{1} 2^{l / r}\left\|\gamma_{v, l} \mid B_{1, \infty}^{\alpha+1}(R)\right\| \cdot\left(M\left|f_{j+l}\right|^{l}\right)^{1 / r}(x) \\
& \leqslant c \sum_{l=-j}^{1} 2^{l / r} 2^{-l \alpha}\left(M\left|f_{j+l}\right|^{1 / r}(x)\right.
\end{aligned}
$$

for any $r, 1 / r<\alpha+1$, with $c$ independent of $j, f$, and $x$. With the aid of (3.17)(3.19) one can follow [6], formulas (4.12)-(4.17) to obtain

$$
\left\|\left(\sum_{v=1}^{\infty} v^{s q-1}\left|\left(f-(C, \alpha)_{v} f\right)(x)\right|^{q}\right)^{1 / q}\left|L_{p}\|\leqslant c\| f\right| F_{p, q}^{s}(T)\right\|
$$

if $1 / \min (1, p, q)<1 / r<\alpha+1, s>0$, and $1 / r-\alpha-s>0$.

This is possible for all $s<1$ (choose $r$ such that $\alpha+1-1 / r$ is sufficiently small).

This proves one direction of the equivalence in (i). The way of obtaining the other direction is described in [6], Theorem 1 and Remark 3. We omit the details.

The proofs of (ii) and (iii) are again consequences of our basic inequality (3.19). One has to modify the above proof in the same way as in [6], Theorems 2, 4, Corollary 3, Remark 6 and 12.

The proof is complete.

\section{References}

[1] J. J. Gergen, Summability of double Fourier series, Duke Math. J. 3 (1937), 133-148.

[2] L. Leindler, Strong Approximation by Fourier Series, Akademiai Kiadó, Budapest 1985.

[3] P. Oswald, On Besov-Hardy-Sobolev spaces of analytic functions in the unit disc, Czechoslovak Math. J. 33 (108) (1983), 408-426.

[4] H.-J. Schmeisser, Characterization of Periodic Function Spaces of Besov-Sobolev Type via Approximation Processes and Relations to the Strong Summability of Fourier Series, this issue, pp. $341-361$.

[5] H.-J. Schmeisser and W. Sickel, On strong summability and smoothness properties of functions, Anal. Math. 8 (1982), 57-70.

[6] -, -, On strong summability of multiple Fourier series and approximation of periodic functions, Math. Nachr. 133 (1987), 211-236.

[7] -, -, On approximation by Riesz and Abel-Poisson means in periodic Besov-TriebelLizorkin spaces. Seminar Analysis 1986/87, Teubner-Texte Math., Bd. 106, Teubner, Leipzig 1988.

[8] H.-J. Schmeisser and H. Triebel, Topics in Fourier Analysis and Function Spaces, Akad. Verlagsgesellschaft Geest \& Portig K.-G., Leipzig 1986 and Wiley, Chichester 1987. 
[9] W. Sickel, Periodic spaces and relations to strong summability of multiple Fourier series, Math. Nachr. 124 (1985), 15-44.

[10] W. Trebels, Multipliers for $(C, \alpha)$-Bounded Fourier Expansions in Banach Spaces and Approximation Theory, Lect. Notes in Math. 329, Springer-Verlag, Berlin-Heidelberg-New York, 1973.

[11] H. Triebel, Theory of Function Spaces, Akad. Verlagsgesellschaft Geest \& Portig K.-G., Leipzig, and Birkhăuser Verlag, Basel, Boston, Stuttgart, 1983.

Presented to the Semester

Approximation and Function Spaces

February 27-May 27, 1986 